\section{Workshop über Acrylatchemie}

Mit dem Ziel, das Verständnis für die Möglichkeiten des Einsatzes von Acrylatklebstoffen zu vertiefen, laden die Veranstalter des Kolloquiums „Gemeinsame Forschung in der Klebtechnik“ Klebstoffanwender aus Industrie und Handwerk am 25. Februar 2013 zur Teilnahme am Workshop „Acrylat - Klebstoffchemie für Klebstoffanwender in Industrie und Handwerk" ein.

B evorzugte Einsatzgebiete und besondere Stärken von Klebstoffsystemen, aber auch Einschränkungen der Anwendbarkeit oder spezielle Anforderungen an die zu fügenden Werkstoffe werden ganz wesentlich vom chemischen Charakter der Grundkomponenten festgelegt. Die Kenntnis des Chemismus der Adhäsion, der Aushärtung und der Alterung von Klebsystemen ist nicht nur für den Entwickler, sondern auch für den Anwender von Vorteil, um die Anwendbarkeit auf neuen Gebieten und alternative Klebsys- teme zu beurteilen. Auch bei unerwartetem Verhalten der Klebverbindung unterstützt diese Kenntnis die Problemlösung. Daher laden die Veranstalter des Kolloquiums „Gemeinsame Forschung in der Klebtechnik“ am Vortag des Kolloquiums in loser Reihenfolge zu Workshops mit Themen zur Klebstoffchemie ein. Nach den polyurethanbasierten Systemen in 2011 und den Epoxidharzen in 2012 soll in diesem Jahr im Februar die Chemie der Acrylate nähergebracht werden.

Weitere Infos: www.dechema.de/Acrylat

\title{
GEV verzeichnet regen Mitgliederzuwachs
}

Informationen der Gemeinschaft Emissionskontrollierte Verlegewerkstoffe, Klebstoffe und Bauprodukte e.V. (GEV) zufolge setzt sich der Emicode im europäischen Umland immer weiter durch. Allein innerhalb des letzten Jahres haben sich dem Zertifizierungssystem neun weitere Unternehmen angeschlossen. Damit stieg die Zahl der Hersteller, die ihre Bauwerkstoffe aus dem breiten Marktangebot als sehr emissionsarm („EC 1“ oder „EC 1PLUS“) herausheben möchten, auf über 70 an.

D ie Neuzugänge stammen aus Dänemark, Frankreich, der Türkei, aber auch aus Deutschland. Den meisten Zulauf registrierte die GEV allerdings aus Italien. Mit Carver, Adesiv, der Fila Industria Chimica und Gras Calce schlossen sich gleich vier südeuropäische Bodenverlegewerkstoff-Hersteller dem Emicode-System an und zogen damit landesintern mit Mapei gleich. Der italienische Marktführer war der GEV bereits 2005 beigetreten und gilt hier als Schrittmacher der Entwicklung. „Green-Building-Zertifizierungen sind nicht nur in den USA auf dem Vormarsch und in dieser Hinsicht setzt EC 1PLUS die strengsten Maßstäbe“, erläutert Dr. Uwe Gruber, Geschäftsführer der Mapei GmbH. Mit diesem Trend wachse auch die Nachfrage an Umweltaussagen für Bauprodukte aller Art. Dabei sei der Emicode in Europa das wichtigste Umweltlabel für Verlegewerkstoffe, Klebstoffe und Bauprodukte. Der Beitrag, den der Emicode im dichten Produktdschungel zur Orientierung biete, sei für Hersteller, Verarbeiter und Konsumenten gleichermaßen wertvoll.

\section{Alles rund um Haftklebstoff- Systeme" auf der 37. MKVS}

Um den aktuellen Markttrends Rechnung zu tragen, legten die Veranstalter des 37. Münchener Klebstoff- und VeredelungsSymposiums, das vom 22. bis zum 24. Oktober 2012 im Konferenzzentrum des Sheraton München Arabellapark Hotels stattfand, diesmal den Schwerpunkt auf Haftklebstoff-Systeme.

D ass das Programm aus insgesamt 29 Fachvorträgen zu den aktuellen Trends in der Auftragstechnik und neuen Entwicklungen im Rohstoff- sowie Formulierungsbereich von der Fachwelt bestens aufgenommen wurde, lässt sich u. a. an der hohen Teilnehmerzahl von 280 aus 16 Nationen und der ausgebuchten Table-Top-Ausstellung ablesen.

Besonders hervorzuhebende Highlights waren ein Zwischenbericht über das aktuell laufende Forschungsvorhaben „Haftklebstoffe auf Basis nachwachsender Rohstoffe" vom Karlsruher Institut für Technologie (KIT) und der Übersichtsvortrag „Nachhaltige Anwendungen in der Klebebandindustrie“. Der diesjähriger Plenarvortrag war dem hochaktuelIen Thema „Bioinspirierte wieder ablösbare Klebstoffe" gewidmet.

Das 38. Münchener Klebstoff- und Veredelungs-Symposiums (MKVS) wird vom 28. bis zum 30. Oktober 2013 mit dem Focus „Alles rund um SchmelzklebstoffSysteme“ stattfinden.

Experten auf diesem Gebiet sind bereits jetzt herzlich eingeladen, ihre Abstrakte online einzureichen: www.mkvs.de 